\title{
Excess mortality by patient's multimorbidity, socioeconomic, and healthcare factors, among Diffuse Large B-cell and Follicular lymphomas patients in England: A population- based multilevel study
}

Matthew J. Smith ( $\square$ matthew.smith1@lshtm.ac.uk)

London School of Hygiene \& Tropical Medicine

\section{Aurélien Belot}

London School of Hygiene \& Tropical Medicine

\section{Matteo Quartagno}

University College London

\section{Miguel Angel Luque-Fernandez}

University of Granada

\section{Audrey Bonaventure}

University of Paris

\section{Susan Gachau}

University of Nairobi

\section{Sara Benitez Majano}

London School of Hygiene \& Tropical Medicine

\section{Bernard Rachet}

London School of Hygiene \& Tropical Medicine

Edmund Njeru Njagi

London School of Hygiene \& Tropical Medicine

\section{Research Article}

Keywords: Cancer Epidemiology, Non-Hodgkin Lymphoma, Diffuse Large B-cell Lymphoma, Follicular Lymphoma, Survival Analysis, Comorbidity, Multimorbidity, Socioeconomic Status

Posted Date: February 16th, 2021

DOl: https://doi.org/10.21203/rs.3.rs-209668/v1

License: @ (i) This work is licensed under a Creative Commons Attribution 4.0 International License. Read Full License 


\section{Abstract \\ Background}

Socioeconomic inequalities of survival in patients with non-Hodgkin lymphoma (NHL) persists, which may be explained by patients' comorbidities. We aimed to assess the association between $\mathrm{co} /$ multimorbidity and survival in patients diagnosed with Diffuse Large B-cell (DLBCL) or Follicular lymphoma (FL) in England accounting for other socio-demographic characteristics.

\section{Methods}

Population-based cancer registry data was linked to Hospital Episode Statistics. We used a flexible multilevel excess hazard model to estimate 5 -year net survival and excess mortality by patient's multimorbidity and comorbidity status adjusted for sociodemographic, economic, healthcare factors, and accounting for the patient's area of residence.

\section{Results}

Overall, 15,516 and 29,898 patients were diagnosed with FL and DLBCL in England between 2005-2013, respectively. Respectively, those with comorbidities and multimorbidities had 1.3 (95\% Confidence Interval -Cl-: 1.20-1.40) and 1.5 (95\% $\mathrm{Cl} 1.27-1.87)$ times higher excess mortality compared to those without comorbidities. Patients in most deprived areas showed $26 \%$ (95\% Cl 1.20-1.32) excess mortality risk compared to those in least deprived areas.

\section{Conclusion}

Co/multimorbidities are consistently associated with poorer survival among patients diagnosed with DLBCL or FL. Comorbidities and multimorbidity need to be considered when planning public health interventions targeting haematological malignancies in England.

\section{Key Messages}

- DLBCL and FL patients with comorbidity and multimorbidity show higher excess mortality risk than those without comorbidities in England.

- Comorbidities and multimorbidity are two independent risk factors associated with poorer survival among DLBCL and FL patients after adjusting for socioeconomic status, healthcare factors, and accounting for the patient's area of residence.

- Public health programs targeting the improvement of the survival of DLBCL and FL patients in the most deprived areas in England will have to consider the presence of comorbidities and multimorbidity.

\section{Introduction}

Non-Hodgkin lymphoma (NHL) is a heterogeneous group of malignancies, and is currently the $6^{\text {th }}$ most commonly diagnosed cancer in England: in 2014, approximately 32 males and 23 females per 100,000 people were diagnosed. ${ }^{1}$ Agestandardised mortality rates of males and females are 12 and 8 per 100,000 person-years, respectively. ${ }^{1}$ The heterogeneity in morphology leads to variation in survival probability; for instance, 5-year survival of Follicular Lymphoma (FL) (86.3\%) is higher than Diffuse Large B-cell Lymphoma (DLBCL) (54.8\%). ${ }^{2}$ 
The healthcare system in England aims to offer equitable access to care for all patients. However, variability in health outcomes amongst patients with similar cancers and sociodemographic characteristics still occur, ${ }^{2-4}$ convincing reasons for the variability remains a topic of interest. In 2001, the National Health Service (NHS) Cancer Plan ${ }^{5}$ recognised, and aimed to reduce, the disparities in survival. Since implementation, there is no evidence the Plan had an impact on the inequalities. ${ }^{6,7}$ The deprivation-gap in survival is still apparent, despite the Plan and successive policies, ${ }^{5,8-10}$ illustrating the little understanding of the mechanisms underlying these inequalities and raising the concern that these policies have missed the relevant targets.

Patients' comorbidity status may impact timely diagnosis, possibly leading to treatment with more adverse effects; ${ }^{11}$ comorbidities are, on average, more prevalent and severe amongst more deprived patients. ${ }^{12}$ However, recent evidence indicates that comorbidity explains little of the differential cancer survival between socioeconomic groups. ${ }^{13-15}$ Additionally, research suggests that variations in healthcare access, such as location of residence, could partly explain the inequalities. ${ }^{16-20}$

Overall, the association between comorbidity and survival in patients with DLBCL and FL, accounting for other sociodemographic characteristics and the area of residence, remains unclear. We hypothesize that the presence of comorbidities is associated with poorer survival independently of patients age and socioeconomic status. We aim to study the association between comorbidities and net survival amongst DLBCL or FL patients accounting for sociodemographic and economic factors in England.

\section{Methods}

\section{Study design, participants, data and setting}

We developed a population-based multilevel cohort study of adult patients diagnosed with DLBCL or FL between $1^{\text {st }}$ January 2005 and $31^{\text {st }}$ December 2013 in England. Patients were followed up until death or the end of the study at the $31^{\text {st }}$ December 2015, whichever occurred first.

Information on patients with DLBCL and FL was collected from the linkage of English cancer registry data, the Cancer Analysis System ${ }^{21}$ (CAS) and Hospital Episode Statistics ${ }^{22}$ (HES) datasets within the national cancer registry and analysis service (NCRAS). These datasets contained information on the patient's cancer, subtype (morphology), diagnosis date, comorbidity, admissions, accident and emergency presentations, and outpatient appointments at an NHS hospital.

DLBCL and FL were defined according to the $10^{\text {th }}$ revision of the International Statistical Classification of Diseases and Related Problems (ICD-10 codes C82.0-C85.9) (Supplementary table S2). ${ }^{23}$ Morphology (cell type) and topography (tumour site) were defined using renewed updates of the ICD for Oncology (ICD-O); ICD-O-3 ${ }^{24}$ was used for diagnoses up to 2010 , and ICD-0-3.1 ${ }^{25}$ for diagnoses after 2011.

We obtained the statutory approvals required for this research from the Confidentiality Advisory Group (CAG) of the Health Research Authority (HRA): PIAG 1-05(c) 2007. Ethical approval was obtained from the Research Ethics Committee (REC) of the Health Research Authority (HRA): 07/MRE01/52. Informed consent from participants was waived by the ethics committee. We used anonymised National Cancer Registry and Hospital Episode Statistics data. All methods were carried out in accordance with relevant guidelines and regulations.

\section{Outcome, exposure, and patients' sociodemographic characteristics}


The outcome of the study was the time to death or censoring among DLBCL and FL patients 5 years after cancer diagnosis. We were interested in the estimation of net survival to then derive the excess mortality only due to cancer. Hence, we used England life tables stratified by deprivation, sex, age and calendar year (2005-2013) to account for the overall mortality rate from the background population. ${ }^{26}$

Comorbidity status was the main exposure. We defined comorbidity as the existence of other chronic medical disorders, in addition to cancer, the primary disease of interest, which are causally unrelated to the primary disease. ${ }^{27,28}$ Records from HES were used to identify patients' comorbidity status based on a computational algorithm published elsewhere. ${ }^{29}$ The algorithm seeks for the presence of comorbidities retrospectively and defines a time window of 6 to 24 months prior to cancer diagnosis where comorbidities are not recorded to avoid bias due to the presence of comorbidities related to cancer (i.e., cardiological comorbidities due to DLBCL or FL cancer treatment). Patient's comorbidity status was adapted from the original Charlson comorbidity index ${ }^{30}(\mathrm{CCl})$. We used the Royal College of Surgeons (RCS) modified Charlson Score (Supplementary Table S1). ${ }^{31}$ The score removes patients with a previous malignancy to avoid bias, does not weight differently comorbidities, and categorises comorbidities as: no comorbidities, one comorbidity and two or more comorbidities (multimorbidity).

Socio-demographic and economic characteristics were collected from the HES dataset. Ethnicity was recorded as white or other. Area-level deprivation, classified into one of five quintiles, was determined by the Index of Multiple Deprivation ${ }^{32}$ (IMD), which was based on the Lower Super Output Area $^{33}$ (LSOA) residence of the patient at the time of cancer diagnosis. LSOA is a geographical location with a median of 1500 inhabitants. We also include the information regarding patients' diagnosis path (route to diagnosis), a UK specific programme, classified as: accident and emergency room diagnosis, general practitioner referral (routine and urgent referrals where the patient was not referred under two-week-wait), two-weekwait (urgent GP referral with a suspicion of cancer), and secondary care diagnosis (other outpatient and inpatient elective routes). ${ }^{34}$

\section{Statistical Analysis}

We tabulated the sociodemographic characteristics by DLBCL and FL. To derive patients excess mortality we used a multilevel excess hazard model (EHM) consisting of a smooth function of the baseline hazard ( ) to derive the net survival estimate, and accounting for heterogeneity across LSOAs via the inclusion of a random intercept with mean zero. ${ }^{35}$ The statistical contribution of the random effect to the overall goodness of fit of the model was tested using a likelihood ratio test statistic with a Chi-square mixture distribution. ${ }^{36}$ The was modelled by a cubic B-spline function of time with two knots placed at 1 year and at 3 years after diagnosis where the hazard plateaus.

We include in the model the following variables: age, sex, comorbidities, deprivation, lymphoma subtype, ethnicity, route of cancer diagnosis, and the overall mortality rate from the background population by levels of deprivation, sex, age and calendar year, included as an offset in the model. We included age as a smooth function consisting of a standardized cubic B-spline centred on 70 years and with one knot at the same age. Furthermore, we assumed a time-dependent effect of age at diagnosis, defined as a B-spline function of time, and represented by the interaction between time and age. The parameter estimates for the variables were interpreted conditionally on the random effect, i.e., they have a cluster-specific interpretation, where a cluster refers to a given LSOA. From the model we derived the excess mortality hazard ratios (EMHR) and their respective $95 \%$ confidence intervals $(\mathrm{Cl})$ for all the categorical variables, and the variance of the random effect for the LSOA.

\section{Missing data analysis}


We explored the missing data mechanism for each of the three variables with missing data. Due to clustered data and partially observed categorical variables, we used the latent normal joint modelling multiple imputation approach, under a missing at random assumption (MAR). The imputation model included all fully- and partially-observed variables, vital status indicator, the Nelson-Aalen estimate of the cumulative hazard, and accounted for clustering of patients within lower-super output areas. We generated 10 imputed datasets. The multilevel EHM was fitted to each of these datasets, and results combined using Rubin's rules. ${ }^{37,38}$ Overall tests for the effects of age after multiple imputation were done using the F-based procedure for the test of multiple parameters after multiple imputation. ${ }^{39}$

We used R software for all data analyses; the mexhaz ${ }^{35}$ package was used for excess hazard modelling and the jomo ${ }^{40}$ package for multiple imputation.

\section{Results}

Overall, there were 45,414 patients with $\mathrm{NHL}, 15,516$ (34.2\%) diagnosed with $\mathrm{FL}$ and 29,898 (65.8\%) diagnosed with DLBC in England between 2005 and 2013 (Table 1). Most patients (41,061, 90.4\%) had no comorbidities, while 2,211 (4.9\%) had a comorbidity score of 1 , and $2,142(4.7 \%)$ had a comorbidity score of 2 or more. The prevalence of at least one comorbidity was higher amongst DLBCL (10.7\%) compared to FL (7.5\%). The mean age was 66.2 years overall. The average age was lower amongst FL compared to DLBCL, 63.9 compared to 67.4 years, respectively. The prevalence of DLBCL was higher amongst deprived areas (16.0\%) than FL (14.4\%). 'White' was the most prevalent ethnicity (94.3\%) compared to minority (5.7\%). GP referral was the most common route to diagnosis amongst FL (44.0\%); whereas, amongst DLBCL, A\&E was most common (33.8\%). Variables with missing data were ethnicity (29.6\%) and route to diagnosis (6.8\%).

Table 1: Distribution of cancer subtypes by patient and healthcare system characteristics for patients $(\mathrm{n}=45,414)$ diagnosed with non-Hodgkin lymphoma in England during the period 2005-2013. 
Subtype of NHL

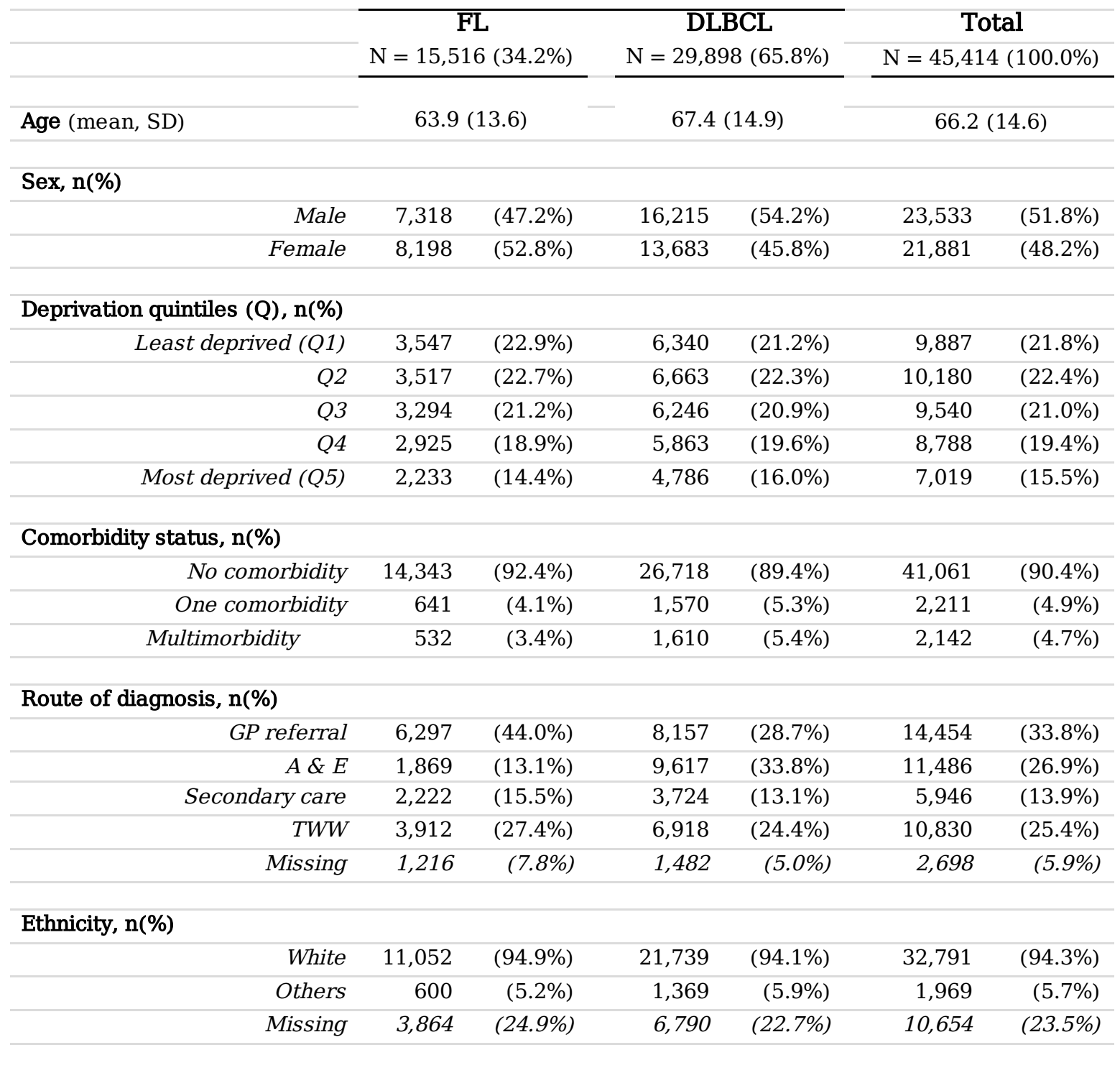

GP: general practitioner referral, A\&E: accident and emergency room, TWW: two-week-wait Complete case analysis: missing ethnicity $23.5 \%$; missing route to diagnosis $5.9 \%$

In the multivariable analysis (Table 2), NHL patients with comorbidity and multimorbidity showed $29 \%$ and $54 \%$ increased excess mortality than patients without comorbidity (i.e., EMHR: 1.29; 95\% Cl: 1.20 - 1.40, and EMHR: 1.54; Cl: 1.27 - 1.87, respectively). Patients living in the most deprived areas had 1.26 (95\% $\mathrm{Cl}: 1.20-1.32)$ times higher excess mortality than those living in the least deprived areas. Patients diagnosed through secondary care showed $21 \%$ increased excess mortality (i.e., EMHR: $1.21 ; 95 \% \mathrm{Cl}: 0.94-1.57$ ) and those diagnoses through the A\&E had nearly three times a higher excess mortality compared to GP referral (i.e., EMHR: 2.72; $95 \% \mathrm{Cl}: 2.46$ - 3.00). Females had a significantly lower excess mortality compared to males (i.e., EMHR 0.92; 95\% Cl: 0.91-0.93). There was, however, no evidence of a difference in excess mortality by ethnicity (Table 2). Using a mixture chi-squared test, there was strong evidence $(p<0.001)$ that including the random effect improved the fit of the model.

Table 2: Adjusted excess mortality hazard ratios adjusted for age, sex, deprivation, comorbidity, cancer subtype, route of diagnosis, ethnicity, and LSOA as random intercept for (i) complete-case analysis, and (ii) after multiple imputation for patients $(\mathrm{n}=45,414)$ diagnosed with non-Hodgkin lymphoma in England during the period 2005-2013. 
Model (i): Complete Case

HR

CI

p-value

Sex

\begin{tabular}{|c|c|c|c|c|c|}
\hline Male & 1.00 & Ref & & 1.00 & Ref \\
\hline Female & 0.92 & $0.88-0.96$ & 0.001 & 0.92 & $0.91-0.93$ \\
\hline
\end{tabular}

Ethnicity

$\begin{array}{rrccccr}\text { White } & 1.00 & \text { Ref } & & 1.00 & \text { Ref } \\ \text { Minorities } & 0.92 & 0.83-1.02 & 0.101 & 0.95 & 0.87-1.03 & 0.230\end{array}$

Deprivation quintiles (Q)

Least deprived $Q 1$

Q2

Q3

Q4

Most deprived Q5

1.00

Ref

1.04

1.10

$0.97-1.12$

$1.02-1.18$

0.279

1.19

$1.11-1.28<0.001$

1.31

$<0.001$

Comorbidity status

No comorbidity $\quad 1.00$

One comorbidity $\quad 1.29$

Multimorbidity

1.60

\section{Ref}

$1.18-1.48<0.001$

$1.47-1.74<0.001$

1.00

1.29

1.54

1.00

1.02

1.08

1.16

1.26

$\begin{array}{cr}\text { Ref } & \\ 0.95-1.09 & 0.662 \\ 1.03-1.13 & 0.003 \\ 1.09-1.23 & <0.001 \\ 1.20-1.32 & <0.001\end{array}$

Lymphoma Subtype

$\begin{array}{rr}F L & 1.00 \\ D L B C L & 2.78\end{array}$

Ref

$2.60-2.97<0.001$

1.00

2.90

Ref

$2.13-3.94<0.001$

Route of diagnosis

$\begin{array}{rr}\text { GP referral } & 1.00 \\ A \& E & 2.65 \\ \text { Secondary Care } & 1.24 \\ T W W & 0.84\end{array}$

Ref

$2.50-2.81<0.001$

$1.15-1.34<0.001$

$0.78-0.90<0.001$

1.00

2.72

1.21

0.84

Ref

$1.20-1.40<0.001$

$1.27-1.87<0.001$

Random Effect

SD (SE) 0.55 (0.05)

$0.48(0.05)$

GP: general practitioner referral. A\&E: accident and emergency room. TWW: two-week-wait

Figure 1 shows the increasing EMHR by age at 3 months, 1- and 5-years (Figure 1A). The EMHR was higher at 3 months compared to 1 or 5 years since diagnosis, particularly among older patients (Figure 1A). However, within the first 6 months after diagnosis, the EMHR of older and younger patients were markedly different compared to those aged 70 years (Figure 1B), which then stabilises, but the increases from 2 years onwards.

Patients without comorbidities had higher net survival compared to those with comorbidity or multimorbidity (Figure 2). Furthermore, patients living in the most deprived areas had a lower net survival at 5 years since diagnosis compared to least deprived areas. The survival gap for deprivation (deprivation-gap) in net survival was apparent from approximately 3 months after diagnosis regardless the comorbidity status.

\section{Discussion}

Comorbidities and multimorbidities are independent risk factors associated with an increased excess mortality risk among DLBCL and FL patients in England. We found strong evidence of a higher excess mortality amongst DLBCL and FL patients diagnosed with comorbidity and multimorbidity compared to patients without comorbidities after adjusting for age, deprivation level, ethnicity, route to diagnosis and accounting for the patient's area of residence. We also found a noticeable deprivation gap in survival, that was consistent regardless of the patient's comorbidity status. 
Differences in access to treatments, or risk of adverse effects, may explain some of the disparities in survival among DLBCL and FL patients. Immunotherapies (rituximab) for the treatment of aggressive lymphomas (e.g. DLBCL) was shown to be effective for those of an advanced age. ${ }^{41-43}$ Rituximab is often used in combination with doxorubicin, an increase in dosage of which is associated with an increased incidence of adverse effects (cardiotoxicity), such as congestive heart failure. ${ }^{44}$ Guidelines based on National Institute for Health and Care Excellence (NICE) and the European Society for Medical Oncology (ESMO) both recommend that patients at risk of cardiotoxicity, or low tolerance of intensive therapy, consider a less-intensive treatment regimen. ${ }^{45-47}$ This less-intensive treatment allocation may partly explain the inequalities in survival by comorbidity status we found.

Evidence shows that the excess mortality among older patients compared to younger patients is highest within the first 3 months and just after 4 years since diagnosis, which could be because of histological transformation from indolence to aggressiveness. Studies suggest the risk of histological transformation increases by $3 \%$ per year. ${ }^{48}$ Therefore, the excess hazard amongst older patients may have increased rather than decreased because histological transformation complexifies the treatment and management of the disease.

The importance of understanding the association of comorbid conditions on cancer patients' outcomes has been well documented. ${ }^{49}$ To our knowledge, this is the first study of England cancer registry data that investigates survival by comorbidity status among DLBCL and FL patients. Our results are consistent with previous findings from a Danish study that showed the hazard of death increased with severity of comorbidity status; 50 however, the Danish study did not account for missing data, and the association with comorbidities was potentially overestimated.

Authors have suggested ways to improve outcomes for patients diagnosed with comorbidities, which include: novel treatment strategies, ${ }^{51}$ inclusion of elderly patients in clinical trials, ${ }^{52,53}$ and investigation of dose-allocation amongst those with higher comorbidity scores. ${ }^{54}$ The deprivation-gap in survival persists even after accounting for prognostic factors such as comorbidity. ${ }^{50,55,56}$ Smith et al. ${ }^{3}$ reported no variation of the deprivation-gap in survival; however, this study may have lacked power, and used an estimator of net survival that is less consistent than other approaches that were readily available at the time of publication. ${ }^{57}$

Consistent with previous studies, ${ }^{4}$ survival after GP referral (non-emergency) diagnosis is significantly better compared to A\&E. However, our study also finds that patients diagnosed through TWW, who would be expected to have worse symptoms and survival, showed no significant difference in survival compared to GP referral. There are two possible reasons for the absence of a difference in the effects. Firstly, GPs could advocate for a prompt referral even though the patient is not on the TWW pathway: resulting in patients with similar access to healthcare facilities. Secondly, on the other hand, patients referred through the TWW pathway have more severe symptoms and expected to have a higher excess hazard. Our results show no difference in the excess mortality indicating that TWW pathway prevents patients with more severe symptoms from having a higher excess hazard: suggesting the performance of TWW pathway is at least as beneficial to a patient's survival as GP referral. However, further factors associated with the interactions between comorbidities and health care systems leading to poorer survival among DLBCL and FL cancer patients need to be studied.

The strengths of this study are that, firstly, we used a large population-based sample size obtained from cancer registry databases linked to HES, which encompasses all patients in England with a diagnosis of DLBCL and FL between 2005 and 2013. Furthermore, we used a latent normal joint modelling multiple imputation to treat missing data in ethnicity and route of diagnosis. . This approach allows imputation of a mix of variable types, while accounting for multilevel structures arising from clustering of patients. ${ }^{39,58,59}$

Survival at 1- and 5-years since diagnosis of DLBCL and FL in England trails that of other European countries; ${ }^{60}$ however, restricting estimates to those surviving at least 1 year after diagnosis (conditional survival) shows a comparable 5-year survival. ${ }^{61}$ This indicates that long-term survival differences are largely explained by the increased short-term mortality. 
Understanding long-term survival in DLBCL and FL is more complex due to the histological transformation of indolent lymphomas, which would require an adaptation of the treatment, support and management from healthcare facilities. This adaptation could be compounded by the patient's susceptibility to cardiotoxic treatments. Further studies could focus on the mechanisms and inequalities of short-term mortality, long-term survival of patients with transformed lymphomas, and survival of patients at risk of cardiotoxicity.

\section{Conclusion}

After accounting for sociodemographic factors, healthcare factors, socioeconomic deprivation and the patient's area of residence, comorbidities and multimorbidity were consistently associated with poorer survival and an increased excess mortality among patients diagnosed with Diffuse Large B-cell (DLBCL) or Follicular lymphoma (FL) in England.

Furthermore, survival inequalities in patients with DLBCL and FL persists between socioeconomic levels after accounting for the presence of comorbidities and multimorbidities. These results show the need for the current framework of the National Health Service to improve the survival of DLBCL and FL patients in the most deprived areas of England, but additionally patient management systems need to consider specific programs for the management of DLBCL and FL patients with comorbidity and multimorbidity.

\section{Declarations}

\section{Acknowledgements}

We would like to thank Adrian Turculet, Data Manager of the LSHTM Inequalities in Cancer Outcomes Network, for his support and assistance with the data linkage.

\section{Author's contributions}

MS, ENN and BR contributed to the conception of the study and designed the study. ENN, ABe, BR, SG, and MQ provided advice on statistical methods. MS conducted the analyses of the data and prepared the draft of the manuscript, tables and figures. ENN, BR and MALF supervised the study and provided comments on the manuscript draft. ENN, BR, MQ, MALF, SG, $\mathrm{SBM}, \mathrm{ABe}$ and $\mathrm{ABo}$ provided comments on the final draft of the manuscript. All authors read and approved the final manuscript.

\section{Declarations}

Funding. This research was funded by Cancer Research UK grant number C7923/A18525. The authors declare no support from any organisations for the submitted work. The design of the study, the analyses and the writing of the manuscript were solely the responsibility of the authors. The findings and conclusions in this manuscript are those of the authors and do not necessarily represent the views of Cancer Research UK.

Availability of data and materials. The data that support the findings of this study are available via application to the Public Health England Office for Data Release, but restrictions apply to the availability of these data.

Ethics approval and consent to participate. We obtained the statutory approvals required for this research from the Confidentiality Advisory Group (CAG) of the Health Research Authority (HRA): PIAG 1-05(c) 2007. Ethical approval was obtained from the Research Ethics Committee (REC) of the Health Research Authority (HRA): 07/MRE01/52. Informed consent from participants was waived by the ethics committee. We used anonymised National Cancer Registry and Hospital Episode Statistics data. All methods were carried out in accordance with relevant guidelines and regulations. 
Competing interests. The authors declare no potential conflicts of interest.

Code availability. Not applicable

\section{References}

1. Smittenaar, C. R., Petersen, K. A., Stewart, K. \& Moitt, N. Cancer incidence and mortality projections in the UK until 2035. Br. J. Cancer. 115, 1147-1155 (2016).

2. Smith, A. et al. Lymphoma incidence, survival and prevalence 2004-2014: sub-type analyses from the UK's Haematological Malignancy Research Network. Br J Cancer. 112, 1575-1584 (2015).

3. Smith, A. et al. Impact of age and socioeconomic status on treatment and survival from aggressive lymphoma: a UK population-based study of diffuse large B-cell lymphoma. Cancer Epidemiol. 39, 1103-1112 (2015).

4. Kane, E. et al. Emergency admission and survival from aggressive non-Hodgkin lymphoma: A report from the UK's population-based Haematological Malignancy Research Network. Eur. J. Cancer. 78, 53-60 (2017).

5. Department of Health. The NHS cancer plan: a plan for investment: a plan for reform. (Department of Health, 2000).

6. Exarchakou, A., Rachet, B., Belot, A., Maringe, C. \& Coleman, M. P. Impact of national cancer policies on cancer survival trends and socioeconomic inequalities in England, 1996-2013: population based study. BMJ. 360, k764-k764 (2018).

7. Maringe, C., Li, R., Mangtani, P., Coleman, M. P. \& Rachet, B. Cancer survival differences between South Asians and nonSouth Asians of England in 1986-2004, accounting for age at diagnosis and deprivation. Br. J. Cancer. 113, 173 (2015).

8. Department of Health. Improving Outcomes: a strategy for cancer. (2011).

9. National Institute for Health and Care Excellence. Improving outcomes in haematological cancers: the manual. (2003).

10. National Institute for Health and Care Excellence. Haematological cancers: improving outcomes. (2016).

11. Renzi, C., Lyratzopoulos, G., Hamilton, W., Maringe, C. \& Rachet, B. Contrasting effects of comorbidities on emergency colon cancer diagnosis: a longitudinal data-linkage study in England. BMC Health Serv. Res. 19, 311 (2019).

12. Fowler, H. et al. Comorbidity prevalence among cancer patients: a population-based cohort study of four cancers. $B M C$ Cancer. 20, 2 (2020).

13. Li, R., Daniel, R. \& Rachet, B. How much do tumor stage and treatment explain socioeconomic inequalities in breast cancer survival? Applying causal mediation analysis to population-based data. Eur. J. Epidemiol. 31, 603-611 (2016).

14. Fowler, H. et al. Persistent inequalities in 90-day colon cancer mortality: an English cohort study. Br. J. Cancer. 117, 1396 (2017).

15. Belot, A. et al. Association between age, deprivation and specific comorbid conditions and the receipt of major surgery in patients with non-small cell lung cancer in England: A population-based study. Thorax 74, 51 LP - 59(2019).

16. Rachet, B. et al. Socioeconomic inequalities in cancer survival in England after the NHS cancer plan. Br J Cancer. 103, 446-453 (2010).

17. Comber, H. et al. Affluence and private health insurance influence treatment and survival in non-Hodgkin's lymphoma.PLoS One11, (2016).

18. Woods, L. M., Rachet, B. \& Coleman, M. P. Origins of socio-economic inequalities in cancer survival: a review. Ann. Oncol. 17, 5-19 (2005).

19. Quaglia, A. et al. Socio-economic factors and health care system characteristics related to cancer survival in the elderly: A population-based analysis in 16 European countries (ELDCARE project). Crit. Rev. Oncol. Hematol. 54, 117-128 (2005).

20. Afshar, N., English, D. R. \& Milne, R. L. Rural-urban residence and cancer survival in high-income countries: A systematic review. Cancer. 125, 2172-2184 (2019).

Page $10 / 14$ 
21. gov.uk. National Cancer Registry and Analysis Service. (2017). Available at: https://www.gov.uk/guidance/nationalcancer-registration-and-analysis-service-ncras. (Accessed: 4th October 2019)

22. NHS Digital. Hospital Episode Statistics. (2015). Available at: 2015. (Accessed: 4th October 2019)

23. International Agency for Research on Cancer. International Classification of Diseases for Oncology. (2013). Available at: http://codes.iarc.fr/. (Accessed: 4th October 2019)

24. Fritz, A. et al. International Classification of Diseases for Oncology(World Health Organisation, 2000).

25. Campo, E. et al. The 2008 WHO classification of lymphoid neoplasms and beyond: evolving concepts and practical applications. Blood. 117, 5019-5032 (2011).

26. Rachet, B. et al. Multivariable flexible modelling for estimating complete, smoothed life tables for sub-national populations. BMC Public Health. 15, 1240 (2015).

27. Porta, M. A Dictionary of Epidemiology(Oxford University Press, 2014). doi:10.1093/acref/9780195314496.001.0001

28. Feinstein, A. R. The pre-therapeutic classification of co-morbidity in chronic disease. J Chronic Dis. 23, 455-468 (1970).

29. Maringe, C., Fowler, H., Rachet, B. \& Luque-Fernandez, M. A. Reproducibility, reliability and validity of population-based administrative health data for the assessment of cancer non-related comorbidities. PLoS One. 12, e0172814 (2017).

30. Charlson, M. E., Pompei, P., Ales, K. L. \& MacKenzie, C. R. A new method of classifying prognostic comorbidity in longitudinal studies: Development and validation. J. Chronic Dis. 40, 373-383 (1987).

31. Armitage, J. N. \& van der Meulen, J. H. Identifying co-morbidity in surgical patients using administrative data with the Royal College of Surgeons Charlson Score. Br J Surg. 97, 772-781 (2010).

32. gov.uk. Indices of Mulitple Deprivation. (2015). Available at: https://www.gov.uk/government/statistics/english-indicesof-deprivation-2015. (Accessed: 4th October 2019)

33. National Health Service: data dictionary. Lower Super Output Area. (2018). Available at:

https://www.datadictionary.nhs.uk/data_dictionary/nhs_business_definitions/l/lower_layer_super_output_area_de.asp? shownav=1. (Accessed: 4th October 2019)

34. Elliss-Brookes, L. et al. Routes to diagnosis for cancer - determining the patient journey using multiple routine data sets. Br J Cancer. 107, 1220-1226 (2012).

35. Charvat, H. et al. A multilevel excess hazard model to estimate net survival on hierarchical data allowing for non-linear and non-proportional effects of covariates. Stat Med. 35, 3066-3084 (2016).

36. Verbeke, G. \& Molenberghs, G. Linear Mixed Models for Longitudinal Data (Springer-Verlag, New York, 2000).

37. Little, R. J. \& Rubin, D. B. Statistical Analysis with Missing Data. (John Wiley \& Sons, Inc 1987).

38. Rubin, D. B. Multiple imputation for nonresponse in surveys(Wiley, 1987).

39. Carpenter, J. R. \& Kenward, M. G. Multiple Imputation and Its Application (John Wiley \& Sons, Ltd, 2013).

40. Quartagno, M. \& Carpenter, J. R. jomo: A package for multilevel joint modeling multiple imputation. (2016).

41. Coiffier, B. et al. CHOP Chemotherapy plus Rituximab Compared with CHOP Alone in Elderly Patients with Diffuse Large-B-Cell Lymphoma. N. Engl. J. Med. 346, 235-242 (2002).

42. Coiffier, B. Rituximab in combination with CHOP improves survival in elderly patients with aggressive non-Hodgkin's lymphoma. Semin Oncol. 29, 18-22 (2002).

43. Delarue, R. et al. Dose-dense rituximab-CHOP compared with standard rituximab-CHOP in elderly patients with diffuse large B-cell lymphoma (the LNH03-6B study): a randomised phase 3 trial. Lancet Oncol. 14, 525-533 (2013).

44. McGowan, J. V. et al. Anthracycline Chemotherapy and Cardiotoxicity. Cardiovasc. Drugs Ther. 31, 63-75 (2017).

45. National Institute for Health and Care Excellence. Non-Hodgkin's lymphoma: diagnosis and management. (National Institute for Health and Care Excellence, 2016).

46. Tilly, H. et al. Diffuse large B-cell lymphoma (DLBCL): ESMO Clinical Practice Guidelines for diagnosis, treatment and follow-up. Ann. Oncol. 26, v116-v125 (2015).

Page $11 / 14$ 
47. Bröckelmann, P. J. et al. Patient and physician preferences for first-line treatment of classical Hodgkin lymphoma in Germany, France and the United Kingdom. Br. J. Haematol. 184, 202-214 (2019).

48. Lossos, I. S. \& Gascoyne, R. D. Transformation of follicular lymphoma. Best Pract. Res. Clin. Haematol. 24, 147-163 (2011).

49. Sogaard, M., Thomsen, R. W., Bossen, K. S., Sorensen, H. T. \& Norgaard, M. The impact of comorbidity on cancer survival: a review. Clin Epidemiol. 5, 3-29 (2013).

50. Frederiksen, B. L., Dalton, S. O., Osler, M., Steding-Jessen, M. \& de Nully Brown, P. Socioeconomic position, treatment, and survival of non-Hodgkin lymphoma in Denmark-a nationwide study. Br J Cancer. 106, 988-995 (2012).

51. Kobayashi, Y. et al. Charlson Comorbidity Index is an independent prognostic factor among elderly patients with diffuse large B-cell lymphoma. J Cancer Res Clin Oncol. 137, 1079-1084 (2011).

52. Saygin, C. et al. Impact of comorbidities on outcomes of elderly patients with diffuse large B-cell lymphoma. Am. J. Hematol. 92, 989-996 (2017).

53. Chihara, D. et al. Management strategies and outcomes for very elderly patients with diffuse large B-cell lymphoma. Cancer. 122, 3145-3151 (2016).

54. Janssen-Heijnen, M. L. et al. A population-based study of severity of comorbidity among patients with non-Hodgkin's lymphoma: prognostic impact independent of International Prognostic Index. Br J Haematol. 129, 597-606 (2005).

55. Rachet, B., Mitry, E., Shah, A., Cooper, N. \& Coleman, M. P. Survival from non-Hodgkin lymphoma in England and Wales up to 2001. Br. J. Cancer. 99, S104-S106 (2008).

56. Bray, C., Morrison, D. S. \& McKay, P. Socio-economic deprivation and survival of non-Hodgkin lymphoma in Scotland. Leuk. Lymphoma. 49, 917-923 (2008).

57. Pohar Perme, M., Estève, J. \& Rachet, B. Analysing population-based cancer survival - settling the controversies. $B M C$ Cancer. 16, 933 (2016).

58. Carpenter, J., Goldstein, H. \& Kenward, M. REALCOM-IMPUTE software for multilevel multiple imputation with mixed response types.J. Stat. Softw.45, (2011).

59. Quartagno, M. \& Carpenter, J. R. Multiple imputation for discrete data: Evaluation of the joint latent normal model. Biom. J. 61, 1003-1019 (2019).

60. Allemani, C. et al. Global surveillance of trends in cancer survival 2000-14 (CONCORD-3): analysis of individual records for $37,513,025$ patients diagnosed with one of 18 cancers from 322 population-based registries in 71 countries. Lancet. 391, 1023-1075 (2018).

61. Thomson, C. S. \& Forman, D. Cancer survival in England and the influence of early diagnosis: what can we learn from recent EUROCARE results? Br. J. Cancer. 101, S102-S109 (2009).

\section{Figures}


A

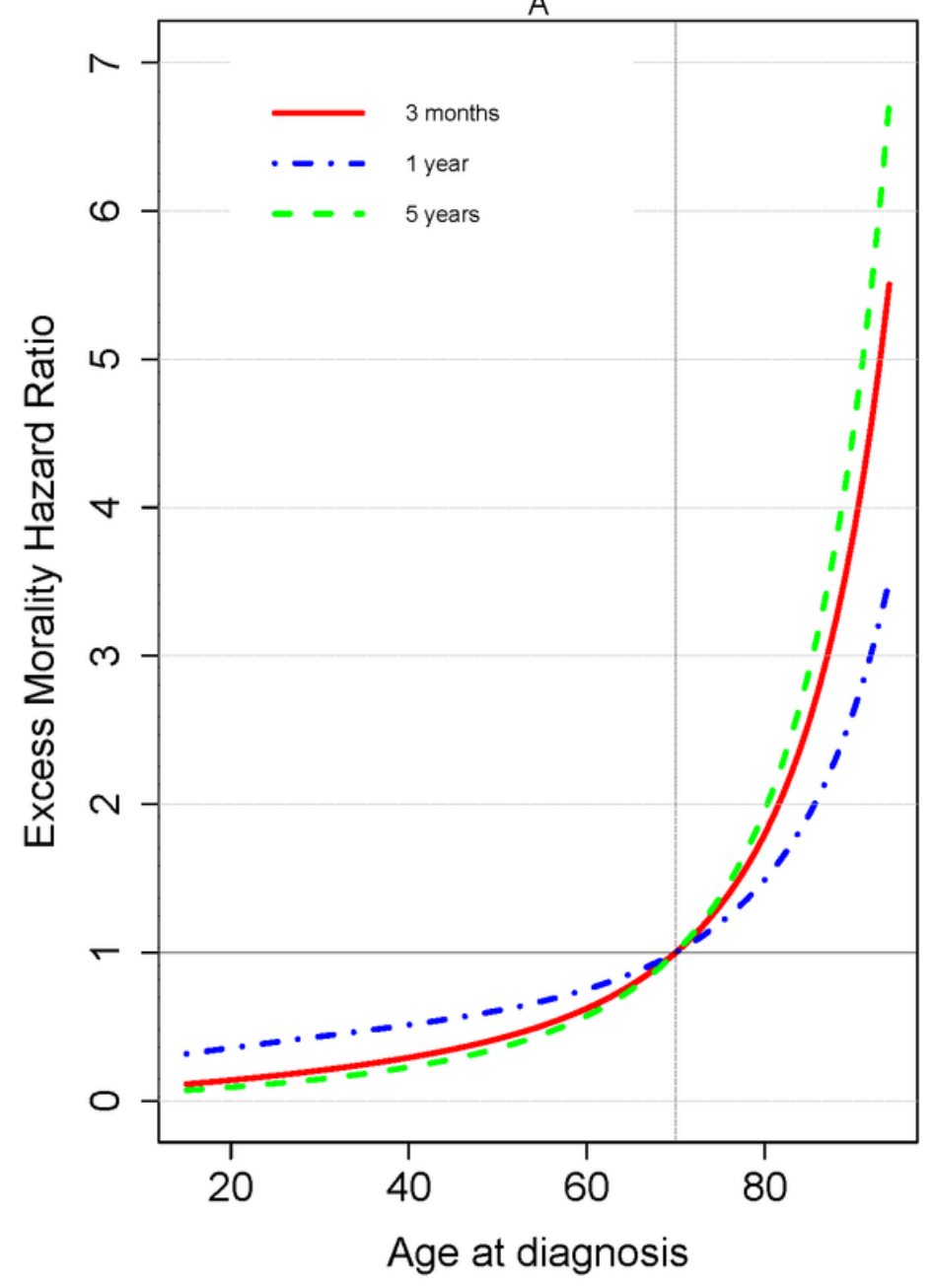

B

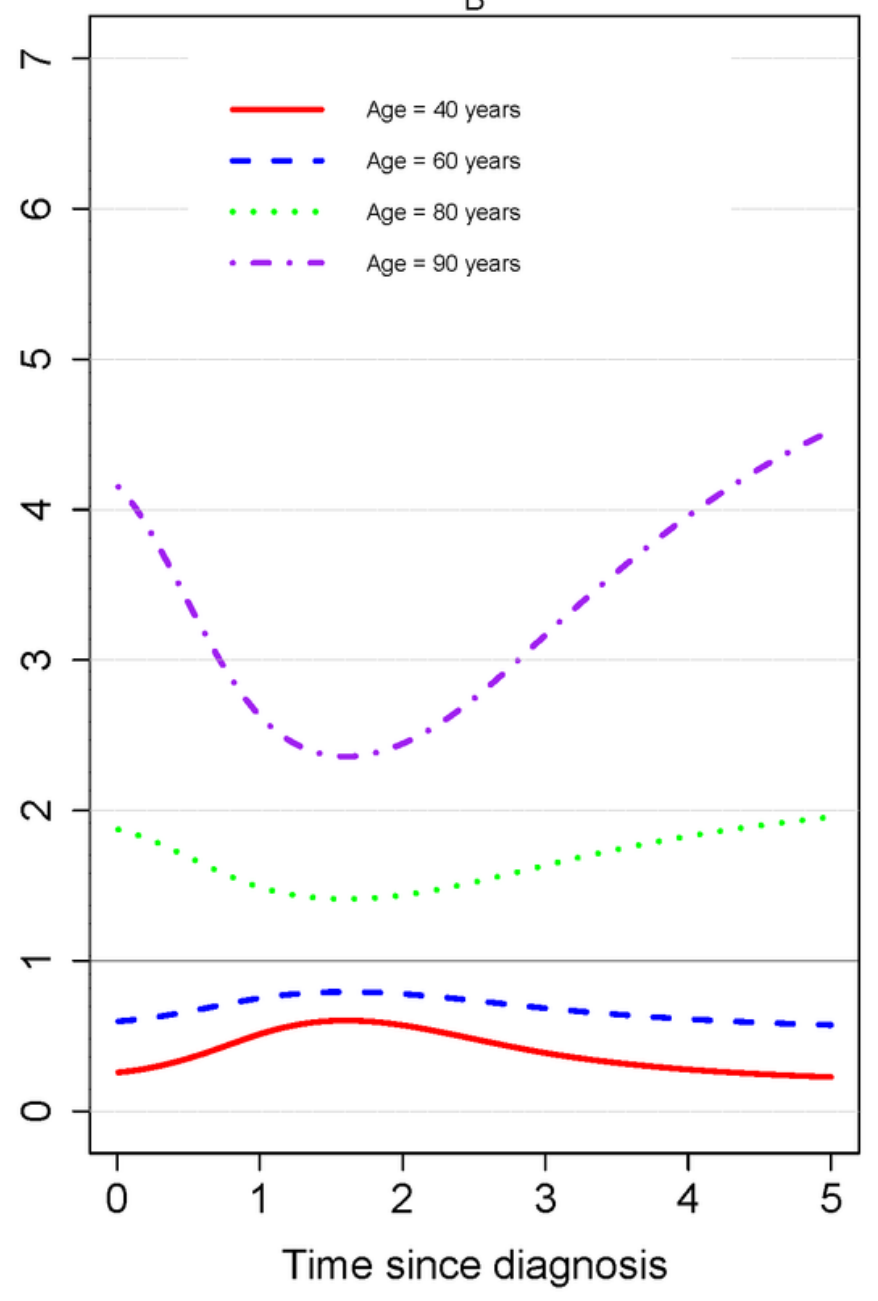

\section{Figure 1}

(A) Excess Mortality hazard ratio (reference 70 years old) for patients diagnosed with non-Hodgkin lymphoma $(n=45,414)$ at 3 months, 1- and 5-years in England during the period 2005-2013 adjusting for all prognostic factors. (B) Survival-time since diagnosis by age groups. 

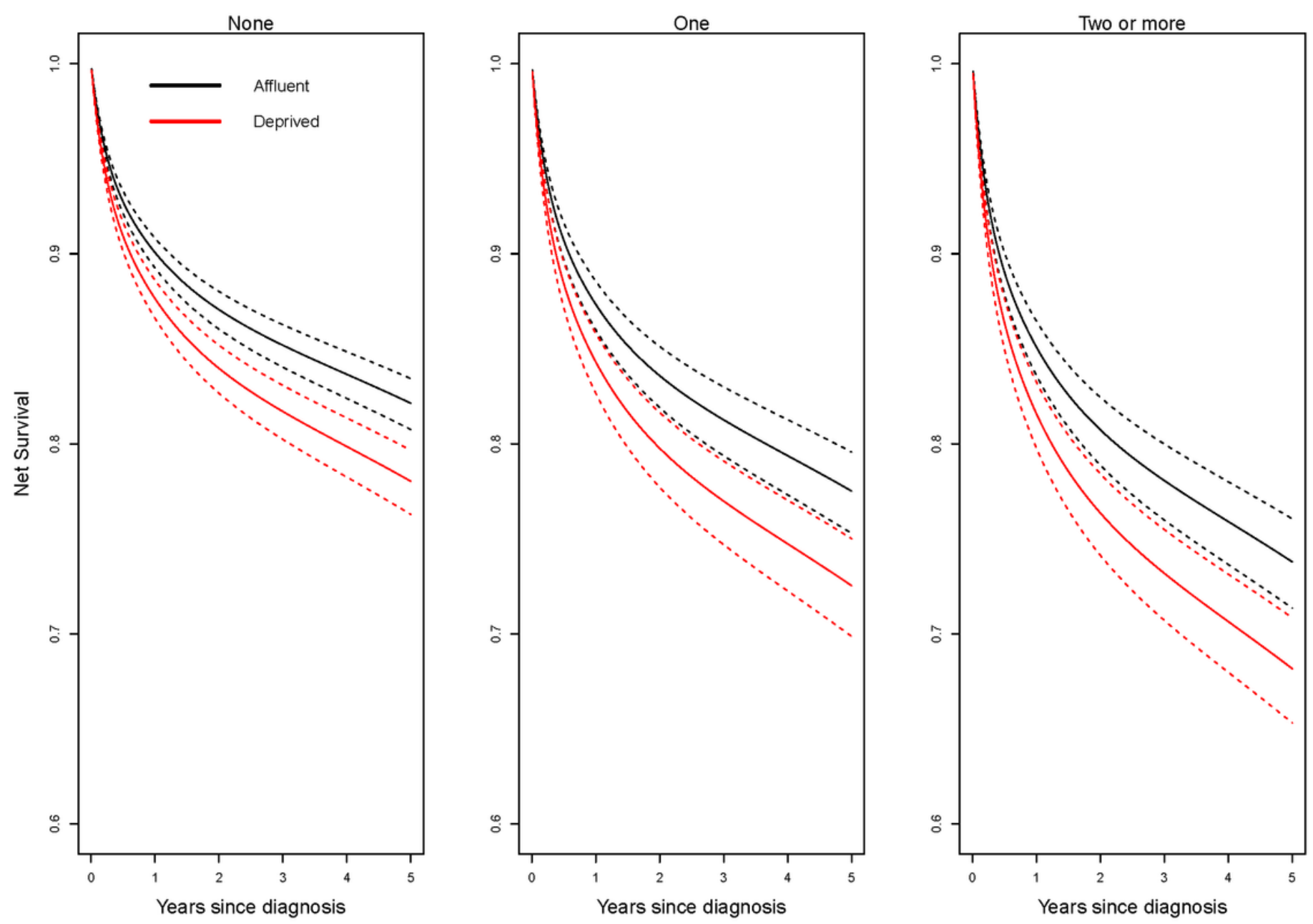

Figure 2

Net survival model-based prediction for non-Hodgkin lymphoma by comorbidity status $(n=45,414)$ in England between 2005-2013.

\section{Supplementary Files}

This is a list of supplementary files associated with this preprint. Click to download.

- Supplementaryfile.docx 